\title{
NeuroImage
}

\section{Cortical thickness analysis in autism with heat kernel smoothing}

\author{
Moo K. Chung, ${ }^{\text {a,b,c, } *}$ Steven M. Robbins, ${ }^{\mathrm{f}}$ Kim M. Dalton, ${ }^{\mathrm{c}}$ Richard J. Davidson, ${ }^{\mathrm{c}, \mathrm{d}}$ \\ Andrew L. Alexander, ${ }^{c, e}$ and Alan C. Evans ${ }^{f}$ \\ ${ }^{a}$ Department of Statistics, University of Wisconsin, Madison, 1210 West Dayton Street, WI 53706, USA \\ ${ }^{\mathrm{b}}$ Biostatistics and Medical Informatics, University of Wisconsin, Madison, 1210 West Dayton Street, WI 53706, USA \\ ${ }^{\mathrm{c}}$ W.M. Keck Laboratory for Functional Brain Imaging and Behavior, University of Wisconsin, Madison, WI 53706, USA \\ ${ }^{\mathrm{d}}$ Department of Psychology and Psychiatry, University of Wisconsin, Madison, WI 53706, USA \\ ${ }^{\mathrm{e}}$ Department of Medical Physics, University of Wisconsin, Madison, WI 53706, USA \\ ${ }^{\mathrm{f}}$ Montreal Neurological Institute, McGill University, Canada
}

Received 5 October 2004; revised 22 November 2004; accepted 3 December 2004

Available online 10 March 2005

\begin{abstract}
We present a novel data smoothing and analysis framework for cortical thickness data defined on the brain cortical manifold. Gaussian kernel smoothing, which weights neighboring observations according to their 3D Euclidean distance, has been widely used in 3D brain images to increase the signal-to-noise ratio. When the observations lie on a convoluted brain surface, however, it is more natural to assign the weights based on the geodesic distance along the surface. We therefore develop a framework for geodesic distance-based kernel smoothing and statistical analysis on the cortical manifolds. As an illustration, we apply our methods in detecting the regions of abnormal cortical thickness in 16 high functioning autistic children via random field based multiple comparison correction that utilizes the new smoothing technique.

(C) 2004 Elsevier Inc. All rights reserved.
\end{abstract}

Keywords: Cortical thickness; Autism; Brain; Heat kernel; Diffusion smoothing

\section{Introduction}

The cerebral cortex is a highly convoluted sheet of gray matter with varying thickness. The pattern of cortical thickness across the cortex varies with different clinical populations (Chung et al., 2003; Kabani et al., 2000). Cortical thickness can be used as an anatomical index to quantify local cortical shape differences. We measure cortical thickness using the inner and outer boundaries of the gray matter. The first step is to obtain T1-weighted magnetic resonance images (MRI) and classify each voxel as one of three tissue types: gray matter, white matter and cerebrospinal fluid (CSF). This classification can be done automatically using, for example, a neural network classifier (Kollakian, 1996) or mixture

\footnotetext{
* Corresponding author. Department of Statistics, University of WisconsinMadison, WI 53706, USA. Fax: +1 6082620032.

E-mail address: mchung@stat.wisc.edu (M.K. Chung).

Available online on ScienceDirect (www.sciencedirect.com).
}

modeling (Ashburner and Friston, 2000). The boundary between gray and white matter voxels gives a crude approximation of the inner cortical boundary which is then refined using a deformable surface algorithm (Dale and Fischl, 1999; Davatzikos and Bryan, 1995; MacDonald et al., 2000). The outer cortical boundary is obtained similarly. In this study we use the method presented in MacDonald et al. (2000) which results in triangular meshes of 40,962 vertices and 81,920 triangles with the average inter-vertex distance of $3 \mathrm{~mm}$ (Fig. 1).

There are many techniques proposed for measuring the cortical thickness from two surface meshes (Fig. 2), for example, the minimum Euclidean distance method of Fischl and Dale (2000), the Laplace equation method of Jones et al. (2000), Bayesian construction of Miller et al. (2000) and the automatic linkage method of MacDonald et al. (2000). In this study, we use the automatic linkage method which has been validated in Kabani et al. (2000) and has been successfully used in Chung et al. (2003) for quantifying normal cortical development.

Before performing cross-subject comparison of spatiallyvarying data, a spatial normalization step is necessary. Spatial normalization consists of spatially transforming each subject anatomy towards a template anatomy. When analyzing 3D datasets such as fMRI, a 3D spatial transformation is used. In this study, however, the data lies on the relatively-thin cortical sheet so a 3D spatial normalization is not applicable: the population of cortices will not generally overlap after normalization in 3D. Instead, we treat each cortex as a surface (using, say, the inner cortical surface) and perform a 2D normalization along the surface. A number of surface registration methods have been published that differ in the measure of discrepancy between the input and template surface, for example, (Dale and Fischl, 1999; Davatzikos, 1997; Fischl et al., 1999; Liu et al., 2004; Robbins, 2003; Thompson and Toga, 1996; Thompson et al., in press; Van Essen et al., 1998). In particular, Davatzikos (1997) uses curvature, Fischl et al. (1999) and Robbins (2003) use some measure of gyrification, while Liu et al. (2004) use attribute 

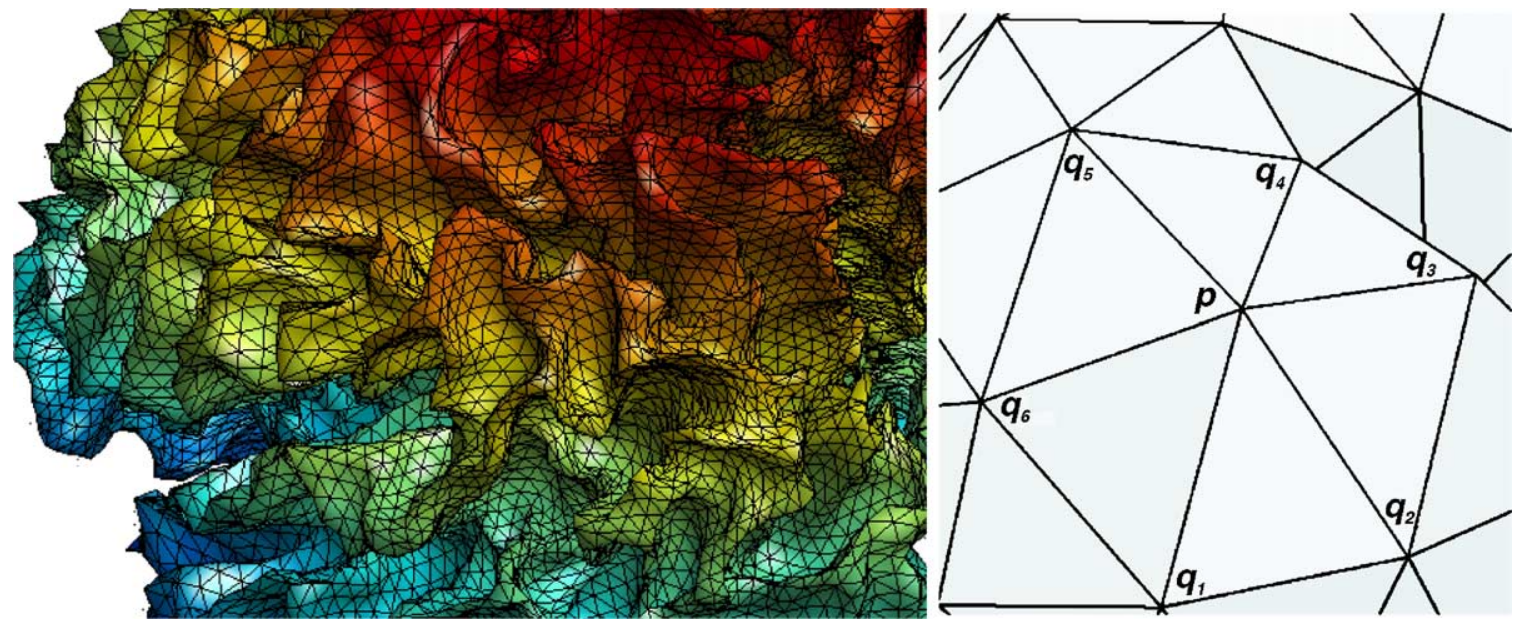

Fig. 1. Left: a typical triangular surface representation of the brain cortical manifold. Right: typical triangular surface with $m=6$ neighboring vertices around $p=q_{0}$.

vectors in trying to minimize the discrepancy. Alternately, Van Essen et al. (1998) and Thompson et al. (in press) solve a partial differential equation that models warping as elastic deformation with landmarks.

Each of the segmentation, thickness computation, and surface registration procedures are expected to introduce noise in the thickness measure. To counteract this, data smoothing is used to increase the signal-to-noise ratio (SNR) and the sensitivity of statistical analysis. For analyzing data in $3 \mathrm{D}$ whole brain images Gaussian kernel smoothing is widely used, which weights neighboring observations according to their 3D Euclidean distance. In this study, however, the data lie on a 2D surface so the smoothing must be weighted according to distance along the surface (Andrade et al., 2001; Chung et al., 2003; Lerch and Evans, 2005; Thompson et al., in press). One such approach, the "anatomically informed basis function" method (Kiebel and Friston, 2002), constructs a non-stationary anisotropic 3D filter kernel in such a way that effectively smoothes functional MRI data along the cortical sheet rather than normal to the sheet. An alternative approach has been developed, known as diffusion smoothing, that smooths data on an explicit 2D cortical surface representation (Andrade et al., 2001; Cachia et al., 2003a; Chung et al., 2003). Diffusion smoothing is based on the observation that, in Euclidean space, Gaussian kernel smoothing is equivalent to solving an isotropic diffusion equation (Koenderink, 1984). This diffusion equation can also be used on the surface manifold (Andrade et al., 2001; Cachia et al., 2003a; Chung et al., 2003), generalizing Gaussian kernel smoothing. The drawback of previous diffusion smoothing methods is the complexity of setting up a finite element method (FEM) for solving the diffusion equation numerically and making the numerical scheme stable. To address this shortcoming, we have developed a simpler method based on heat kernel convolution.

As an illustration, we apply our methods to detect regions of cortical thickness difference between a group of 16 high functioning autistic children and a group of 12 normal children.

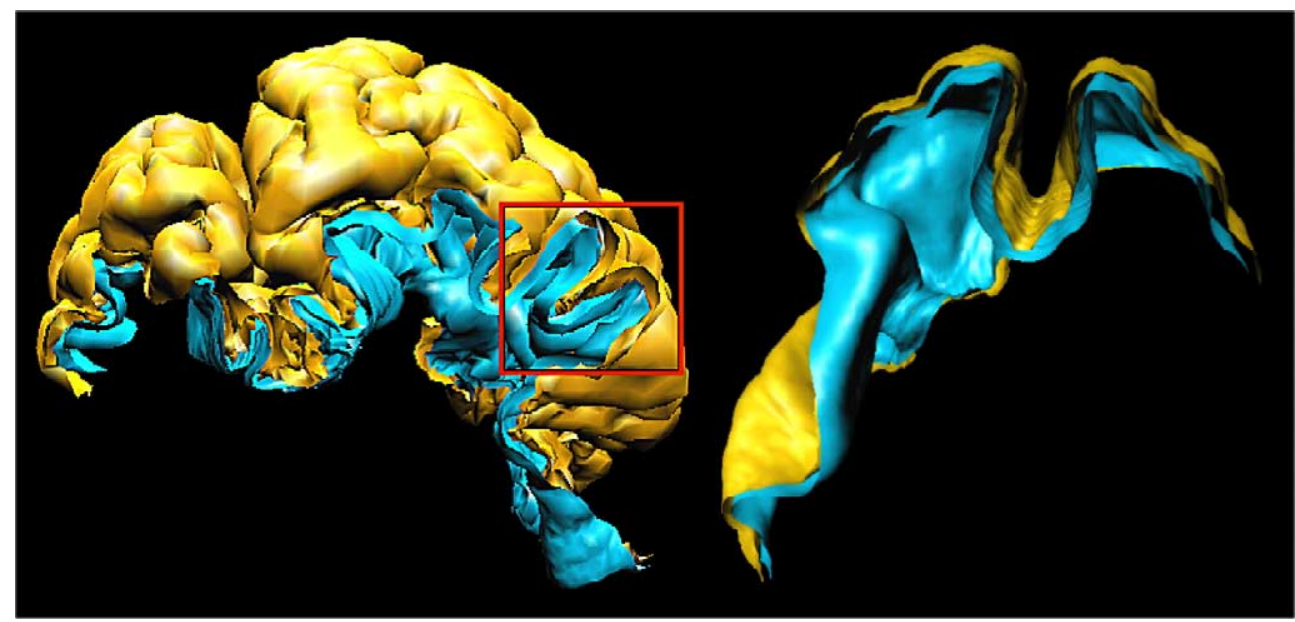

Fig. 2. Anatomy of brain cortex. Left: part of the cortical surface showing both outer (yellow) and inner surface (blue) that bound gray matter. Right: enlargement of the boxed region. The cortical thickness measures the distance between outer and inner surfaces. 
Table 1

Age and relative total gray matter volume distribution $\left(\times 10^{5} \mathrm{~mm}^{3}\right.$ for volume measurements)

\begin{tabular}{|c|c|c|c|c|c|c|c|c|c|c|c|c|c|c|c|c|}
\hline \multicolumn{17}{|l|}{ Control } \\
\hline Age & 15 & 18 & 18 & 16 & 15 & 13 & 18 & 15 & 21 & 17 & 16 & 23 & & & & \\
\hline Volume & 699 & 690 & 704 & 638 & 638 & 671 & 724 & 742 & 701 & 689 & 728 & 714 & & & & \\
\hline \multicolumn{17}{|l|}{ Autistic } \\
\hline Age & 15 & 20 & 17 & 13 & 12 & 15 & 25 & 14 & 15 & 14 & 24 & 18 & 10 & 12 & 22 & 12 \\
\hline Volume & 647 & 725 & 708 & 724 & 776 & 650 & 652 & 661 & 696 & 729 & 672 & 709 & 778 & 781 & 682 & 747 \\
\hline
\end{tabular}

The mean gray matter volumes are $7.08 \pm 0.46 \mathrm{~mm}^{3}$ for the autistic group and $6.95 \pm 0.33 \mathrm{~mm}^{3}$ for the control group. The method for estimating the total gray matter volume is given in Chung et al. (2003).

Regions of statistically significant thickness difference are detected as rejection regions for group comparison tests based on random fields theory.

\section{Subjects and image processing}

Gender and handedness affect brain anatomy (Luders et al., 2003) so all the 16 autistic and 12 control subjects used in this study were screened to be right-handed males except one subject who is ambidextrous. Sixteen autistic subjects were diagnosed with high functioning autism (HFA) via the Autism Diagnostic InterviewRevised (ADI-R) by a trained and certified psychologist at the
Waisman center at the University of Wisconsin-Madison. Twelve healthy, typically developing males with no current or past psychological diagnoses served as a control group. The average age of the control subjects is $17.1 \pm 2.8$ and the autistic subjects is $16.1 \pm 4.5$, which are compatible. The age distribution and relative total gray matter volume for subjects are given in Table 1. The method for estimating the total gray matter volume is given in Chung et al. (2003).

High-resolution anatomical magnetic resonance images (MRI) were obtained using a 3-Tesla GE SIGNA (General Electric Medical Systems, Waukesha, WI) scanner with a quadrature head RF coil. A three-dimensional, spoiled gradient-echo (SPGR) pulse sequence was used to generate $T_{1}$-weighted images. The imaging
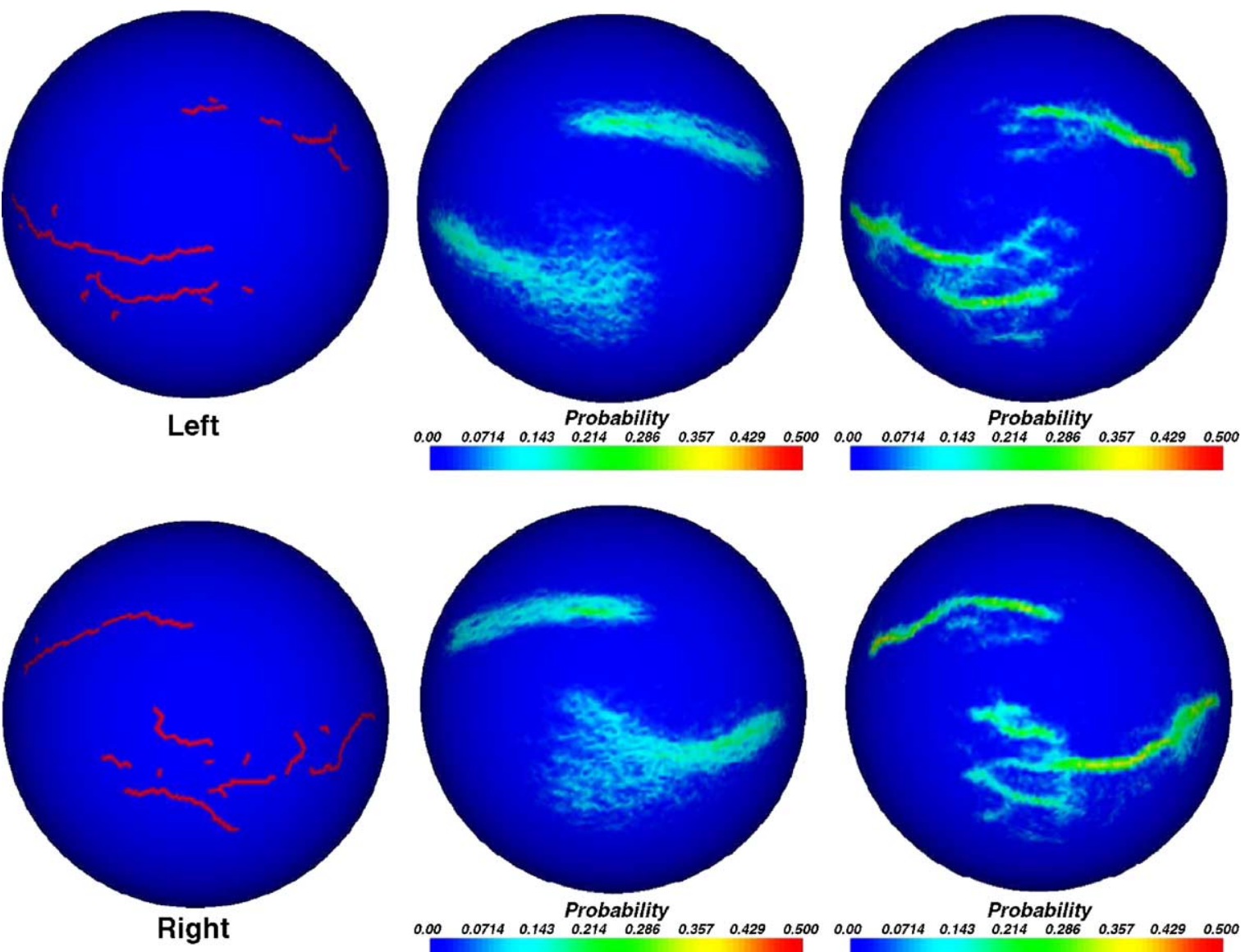

Fig. 3. Automatically generated traces of the central and superior temporal sulcal fundi (Cachia et al., 2003b). The first column shows the traces generated for the template surface. The second column shows the probability of sulcal matching based on 149 normal subjects before any surface normalization. The third column shows the probabilities after surface normalization. The first row is the left hemisphere and the second row is the right hemisphere. Note that the distribution is much more spatially concentrated and the probabilities are much greater after normalization. 

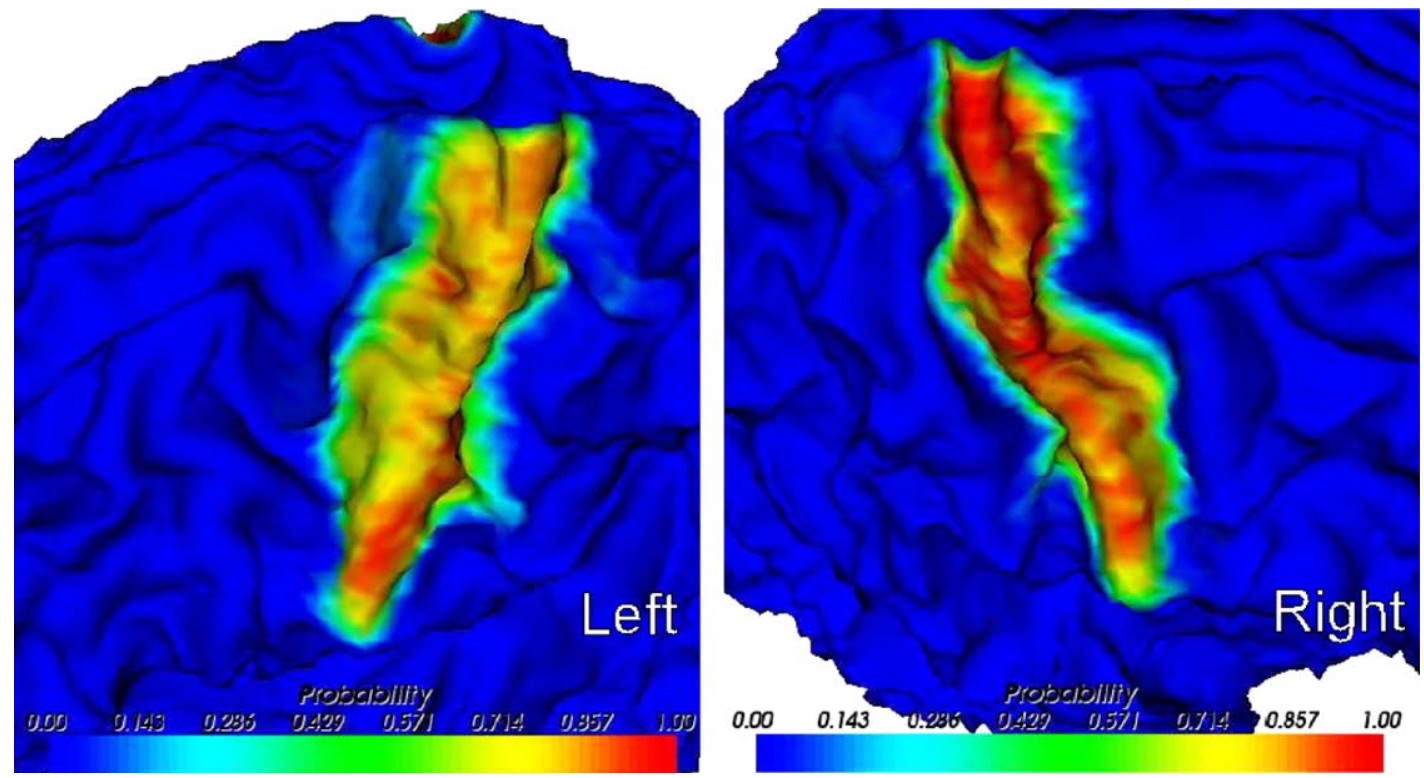

Fig. 4. Probability of sulcal matching, after normalization, for 39 manually identified central sulci defined as the surface region surrounded by gyri, not just the fundus. The views are illustrated on a slightly-opened version of the template cortical surface in order to better view inside the sulcus. The warping in $2 \mathrm{D}$ localizes the central sulcus nearly completely inside the template central sulcus. Left (right) figure is the left (right) central sulci.

parameters were $\mathrm{TR} / \mathrm{TE}=21 / 8 \mathrm{~ms}$, flip angle $=30^{\circ}, 240 \mathrm{~mm}$ field of view, $256 \times 192$ in-plane acquisition matrix (interpolated on the scanner to $256 \times 256)$, and 128 axial slices $(1.2 \mathrm{~mm}$ thick $)$ covering the whole brain.

Each $T_{1}$-weighted image subsequently underwent several image preprocessing steps. Image intensity nonuniformity was corrected using nonparametric nonuniform intensity normalization method (N3), which eliminates the dependence of the field estimate on anatomy (Sled et al., 1988). Then, using the automatic image processing pipeline (Zijdenbos et al., 1998), the image was spatially normalized into the MNI stereotaxic space using a global affine transformation (Collins et al., 1994). Subsequently, an automatic tissue-segmentation algorithm based on a supervised artificial neural network classifier was used to classify each voxel as cerebrospinal fluid (CSF), gray matter, or white matter (Kollakian, 1996). Brain substructures such as the brain stem and the cerebellum were removed automatically. Triangular meshes for inner and outer cortical surfaces were obtained by a

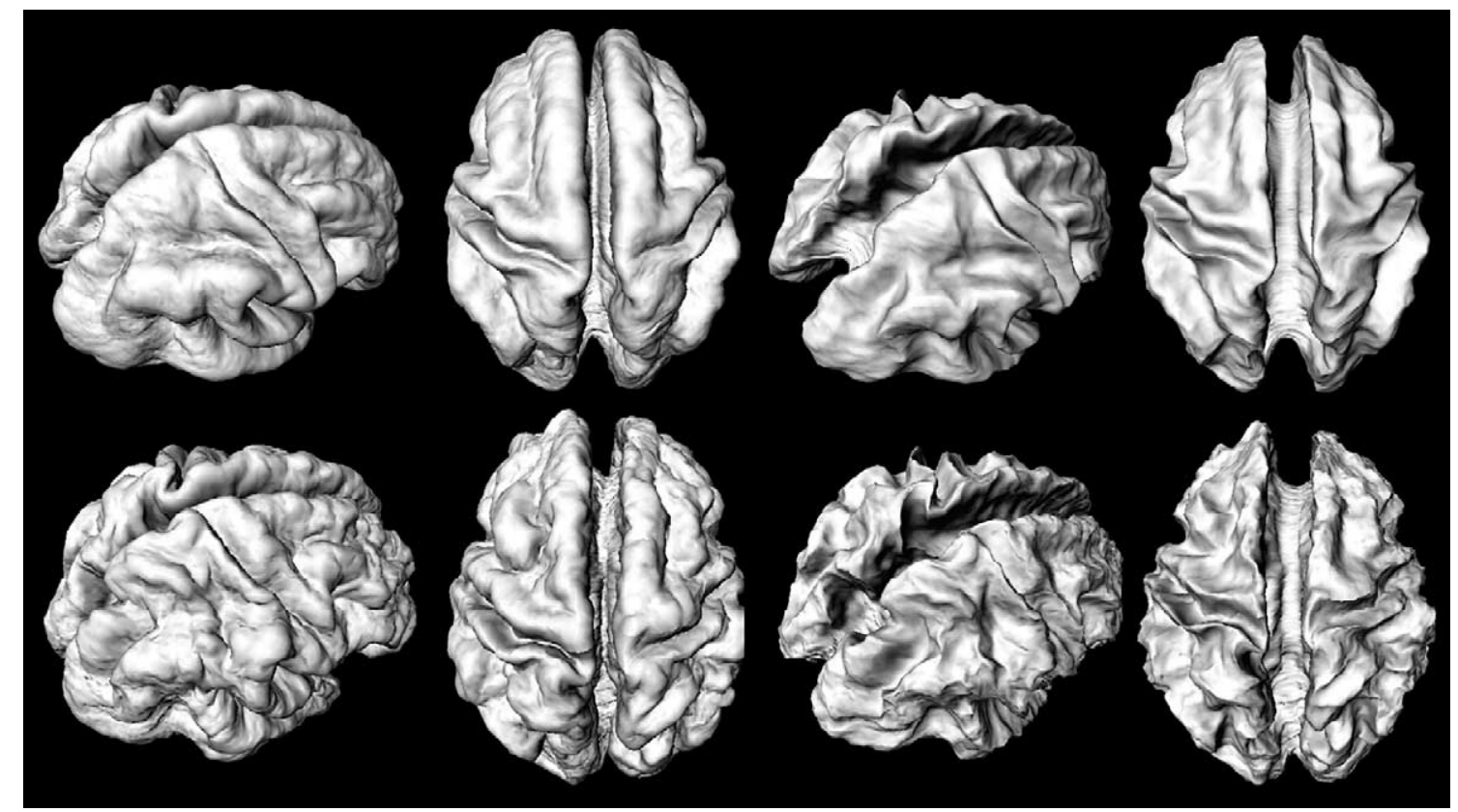

Fig. 5. Top: average surface of the normal subjects, on which statistical maps are projected, constructed via the surface registration method in Chung et al. (2003). First two images show the outer cortical surface and the next two images show the inner cortical surface. Bottom: average template constructed via the improved surface warping method providing more detailed anatomy. 
deformable surface algorithm (MacDonald et al., 2000). Such a deformable surface approach has the advantage that the surface topology can be fixed to be spherical and the deformation process can maintain a non-intersecting surface at all times (MacDonald et al., 2000), obviating the need for topology correction (Dale and Fischl, 1999; Liu et al., 2004). The mesh starts as an ellipsoid located outside the brain and is shrunk to obtain the inner cortical surface. Then, the inner surface is expanded, with constraints, to obtain the outer cortical surface. These procedures result in triangular mesh representation of the cortical surfaces consisting of 40,962 vertices and 81,920 triangles with an average edge length of $3 \mathrm{~mm}$ (Fig. 1 and 2). The triangular meshes are not constrained to lie on voxel boundaries. Instead, the triangular meshes can cut through a voxel, which serves to reduce discretization error and partial volume effect.

Thickness is measured using the natural anatomical homology between vertices on the inner and outer cortical surface meshes, since the outer surface is obtained by deforming the inner surface. The distance between corresponding vertices on both surfaces is the measurement used here as the cortical thickness (MacDonald et al., 2000). The methodology has been validated in Kabani et al. (2000) and has been used in Chung et al. (2003) and Lerch and Evans (2005).

Spatial normalization of the surfaces is necessary to facilitate vertex-by-vertex inter-subject thickness comparison. Our spatial normalization obtains a 2D warping from each inner brain surface (which is easier to detect on a typical MRI than the outer surface) to a template inner brain surface. The surface deformation field from one surface to the template surface is obtained by minimizing an objective function that measures the global fit of two surfaces, while maximizing the smoothness of the deformation in such a way that the pattern of gyral ridges are matched smoothly. This regularization mechanism produces a smooth deformation field, with very little folding. The deformation field is parameterized using a triangulated mesh and the algorithm proceeds in a coarseto-fine manner, with four levels of mesh resolution. This surface registration method has been validated in Robbins (2003). Figs. 3 and 4 illustrate the effectiveness of this algorithm by computing the probability of matching superior temporal sulcal fundi and central sulci. Fig. 5 demonstrates the improvement of our new surface normalization over the previous approach in Chung et al. (2003). The top images in Fig. 5 are the average surfaces of the normal subjects constructed using the previous approach in Chung et al. (2003), while the bottom images are based on our new improved surface warping method showing more detailed anatomy.

\section{Heat kernel smoothing}

All the preceding image accession and processing steps introduce unwanted noise into the cortical thickness measurements. Consider the following stochastic model for thickness measurement on cortical manifold $\partial \Omega$ :

$Y(p)=\theta(p)+\varepsilon(p), p \in \partial \Omega$

where $\theta$ is the mean thickness and $\varepsilon$ is a zero mean Gaussian random field. The cortical surface $\partial \Omega$ can be assumed to be a smooth 2-dimensional Riemannian manifolds (Dale and Fischl, 1999; Joshi et al., 1995). We define the heat kernel smoothing estimator of data $\theta$ to be the convolution

$\hat{\theta}(p)=K_{\sigma} \times Y(p)=\int_{\partial \Omega} K_{\sigma}(p, q) Y(q) \mathrm{d} \mu(q)$

where $\mu(q)$ is a surface measure (the Lebesgue measure unless otherwise stated), $\sigma$ is the smoothing parameter (band width), $K_{\sigma}$ is the heat kernel given in terms of the eigenfunctions of the Laplace-Beltrami operator. For an overview of heat kernel and its spectral representation, one may refer to Rosenberg (1997) and Berline et al. (1991). For our study, it is sufficient to note that the heat kernel converges to a Gaussian kernel locally as the band width $\sigma$ goes to zero. More rigorous mathematical formulation can be found in Chung (2004). Convolution (2) can be viewed as the minimizer of the weighted least-squares errors in a regression setting. Let us list a couple of important properties of heat kernel smoothing.

Property 1. $K_{\sigma} \times Y$ is the unique solution of the following initial value problem at time $t=\sigma^{2} / 2$ :

$\frac{\partial f}{\partial t}=\Delta f, f(p, 0)=Y(p)$

where $\Delta$ is the Laplace-Beltrami operator on $\partial \Omega$.

This is a well-known result in differential geometry (Rosenberg, 1997). In Andrade et al. (2001) and Cachia et al. (2003a), smoothing is done by solving the heat equation via the combination of the least squares estimation of the LaplaceBeltrami operator and the finite difference method (FDM). In Chung et al. (2003), the heat Eq. (3) is solved using the finite element method (FEM) and a similar FDM. The problem with these approaches to data smoothing is the complexity of setting up the FEM and making the FDM converge (Cachia et al., 2003a; Chung and Taylor, 2004). Our proposed heat kernel smoothing will avoid all these problems.

Let $d(p, q)$ denote the geodesic distance between points $p$ and $q$ of the cortical manifold. A covariance function $R$ on the manifold is said to be isotropic if it is a function only of the geodesic distance, that is, $R(p, q)=\rho(d(p, q))$ for some function $\rho$.

Property 2. Suppose the covariance function of $Y$ in Eq. (1) is a decreasing isotropic function, that is, of form $R_{Y}(p, q)=\rho(d(p, q))$ with $\rho$ decreasing. Then,

$\operatorname{Var}\left[K_{\sigma} \times Y(p)\right] \leq \operatorname{Var} Y(p)$ for each $p \in \partial \Omega$.

Hence, heat kernel smoothing reduces the between-subject variability of thickness measurements. This is illustrated in the right figure in Fig. 7, where it shows the decreasing variance over the number of iterations for 10 simulated thickness data.

\section{Property 3.}

$\lim _{\sigma \rightarrow \infty} K_{\sigma} \times Y=\frac{\int_{\partial \Omega} Y(q) \mathrm{d} \mu(q)}{\mu(\partial \Omega)}$.

A similar result for heat kernel is given in Rosenberg (1997). When we choose a large band width, heat kernel smoothing converges to the sample mean of data on a cortical manifold. This is illustrated in Fig. 6. It shows increasing convergence to the within-subject mean thickness as the number of iterations 


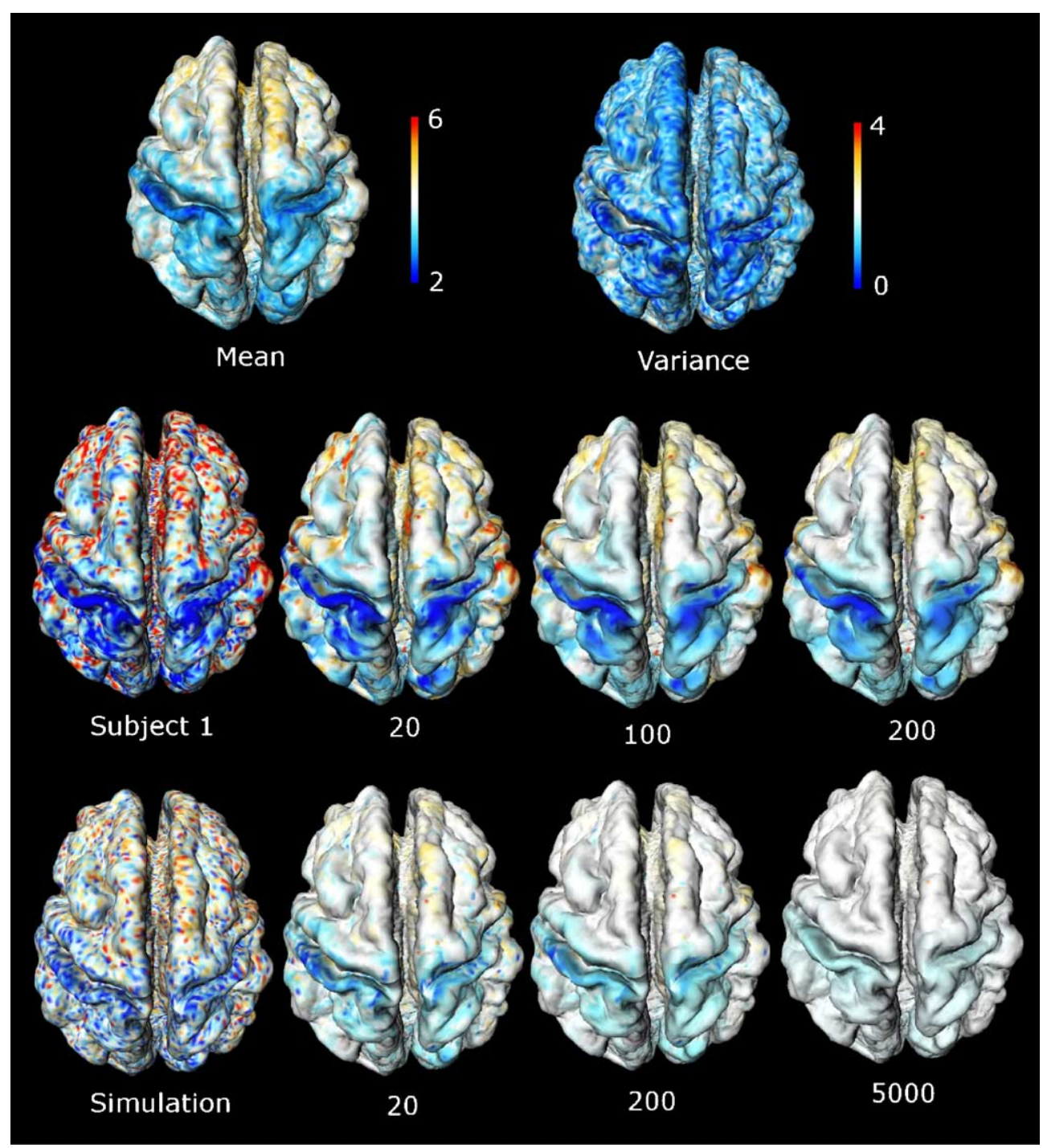

Fig. 6. Heat kernel smoothing on real and simulated data. Top: the sample mean and the sample variance of 12 normal subjects. These are used in generating simulated data. Middle: iterated heat kernel smoothing of real data with $\sigma=1$ and $k=20,100,200$. Bottom: iterated heat kernel smoothing of simulated data with $\sigma=1$ and $k=20,200,5000$. At $k=5000$ iterations, it shows the increasing convergence to the within-subject mean thickness (Property 3 ).

increases. Fig. 7 also shows the decreasing within-subject variance over the number of iterations demonstrating that the heat kernel smoothing converges to a constant, namely the within-subject mean thickness.

Property 4. Heat kernel smoothing with large band width can be decomposed into iterated kernel smoothing with smaller band width via

$$
K_{\sigma}^{(k)} \times f=\underbrace{K_{\sigma} \times \ldots \times K_{\sigma}}_{k \text { times }} \times f=K_{\sqrt{k} \sigma} \times f .
$$

This can be seen as the scale space property of diffusion. From Property $1, K_{\sigma} \times\left(K_{\sigma} \times Y\right)$ can be taken as the diffusion of signal $K_{\sigma} \times Y$ after time $\sigma^{2} / 2$ so that $K_{\sigma} \times\left(K_{\sigma} \times Y\right)$ is the diffusion of signal $Y$ after time $\sigma^{2}$. Hence

$$
K_{\sigma} \times K_{\sigma} \times Y=K_{\sqrt{2} \sigma} \times Y .
$$

Arguing inductively, we see that the general statement holds. This is the basis of our heat kernel smoothing formulation. By decomposing a smoothing of large band width into iterated smoothing each using a smaller band width, we can construct the heat kernel in a much smaller domain. For numerical implementation, we use an asymptotic representation called the parametrix expansion (Rosenberg, 1997):

$K_{\sigma}(p, q)=\frac{1}{(2 \pi \sigma)^{1 / 2}} \exp \left[-\frac{d^{2}(p, q)}{2 \sigma^{2}}\right]\left[u_{0}(p, q)+O\left(\sigma^{2}\right)\right]$

The first term $u_{0}(p, q) \rightarrow 1$ as $p \rightarrow q$ and the manifold becomes flat. Assuming that $\sigma$ is sufficiently small and $q$ is sufficiently close to $p$, we have

$K_{\sigma}(p, q) \approx \frac{1}{(2 \pi \sigma)^{1 / 2}} \exp \left[-\frac{d^{2}(p, q)}{2 \sigma^{2}}\right]$

For the case at hand, we have cortical thickness measurements only at surface mesh vertices so it is natural to use a discrete version of convolution. Let $q_{1}, \ldots, q_{m}$ be neighboring vertices of $p=q_{0}$ (see 

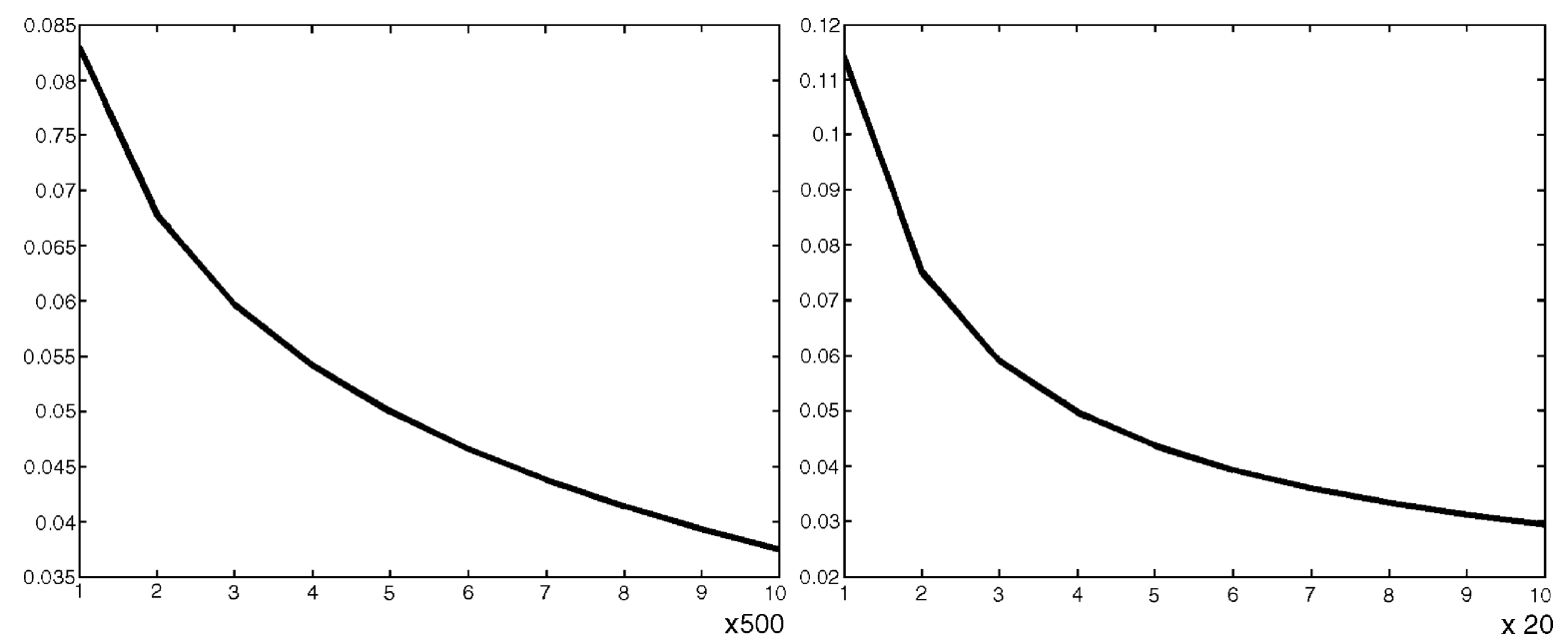

Fig. 7. Left: within-subject variance plotted over the number of iterations of heat kernel smoothing. Decreasing variance implies the convergence of the heat kernel smoothing to the within-subject mean (Property 3). Right: between-subject variance plotted over the number of iterations illustrating Property 2.

Fig. 1) and let $N_{p}=\left\{q_{0}, q_{1}, \ldots, q_{m}\right\}$. The geodesic distance between adjacent vertices is the $3 \mathrm{D}$ Euclidean distance, that is, $d\left(p, q_{i}\right)=$ $\left\|p-q_{i}\right\|$. We define the normalized truncated kernel for a polygonal surface to be

$\widetilde{W}_{\sigma}\left(p, q_{i}\right)=\frac{\exp \left[-\frac{\mathrm{d}^{2}\left(p, q_{i}\right)}{2 \sigma^{2}}\right]}{\sum_{j=0}^{m} \exp \left[-\frac{\mathrm{d}^{2}\left(p, q_{j}\right)}{2 \sigma^{2}}\right]}$.

The discrete convolution is defined by

$\widetilde{W}_{\sigma} \times Y(p)=\sum_{i=0}^{m} \widetilde{W}_{\sigma}\left(p, q_{i}\right) Y\left(q_{i}\right)$

Note that $\widetilde{W}_{\sigma}$ is a discrete probability distribution, that is, $\sum_{i=0}^{m}$ $\widetilde{W}_{\sigma}\left(p, q_{i}\right)=1$. Let polygonal surface $S$ have $n$ vertices $p_{1}, \ldots, p_{n}$. Then we have the following algorithm ${ }^{1}$ for the heat kernel smoothing.

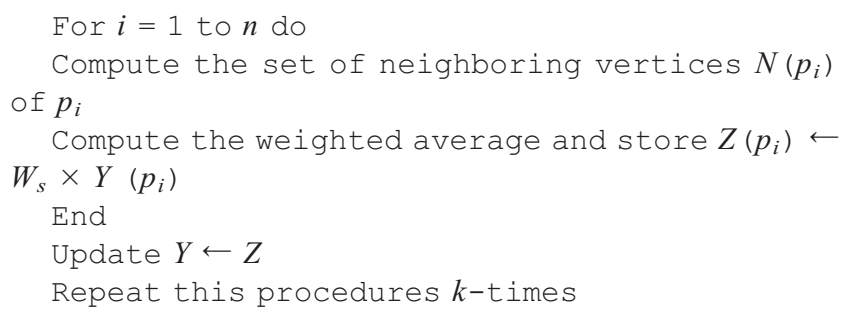

Using the above algorithm, we performed a simulation study. First, we computed the sample mean $\bar{\theta}(p)$ and the sample variance $S^{2}(p)$ of the cortical thickness for 12 normal subjects at each vertex $p$. The resulting mean and variance maps are given in Fig. 6 . Then, we generated 10 Gaussian random numbers with mean $\bar{\theta}(p)$ and variance $S^{2}(p)$ at each vertex, rejecting any value smaller than 2 or larger than 6 to make the simulation more realistic. This procedure generated 10 simulated cortical thickness data sets. Then we performed heat kernel smoothing with $\sigma=1$. Fig. 6 shows the process of smoothing at iteration $k=20,100,200$ for one real data

\footnotetext{
1 The MATLAB code for heat kernel smoothing is freely available at http://www.stat.wisc.edu/ mohung/softwares/hk/hk.html.
}

set and $k=20,200,5000$ for one simulated data set. As the number of iterations increase the heat kernel smoothing converges to the within-subject mean, demonstrating Property 3. This is further demonstrated by plotting the decreasing variance over the number of iterations in Fig. 7. We also plotted between-subject variance over the number of iterations in Fig. 7, demonstrating Property 2.

\section{Statistical analysis on cortical manifolds}

The first group consists of autistic subjects and the second group consists of normal control. For the $i$ th group, let $n_{i}$ denote the number of subjects and $\theta i$ denote the population mean thickness. Under stochastic model (1), we are interested in testing if the thickness for the two groups are identical, that is,

$H_{0}: \theta_{1}(p)=\theta_{2}(p)$ for all $p \in \partial \Omega$

vs.

$H_{1}: \theta_{1}(p)>\theta_{2}(p)$ for some $p \in \partial \Omega$.

The above null hypothesis is the intersection of the collection of hypotheses

$H_{0}=\bigcap_{p \in \partial \Omega} H_{0}(p)$

where $H_{0}(p): \theta_{1}(p)=\theta_{2}(p)$. Assuming the variability of the two groups to be same, the test statistic to use is the two-sample $t$ statistic with equal variance given by

$T(p)=\frac{\bar{\theta}_{1}-\theta_{1}-\left(\bar{\theta}_{2}-\theta_{2}\right)}{S_{p} \sqrt{1 / n_{1}+1 / n_{2}}}$

where the pooled variance $S_{p}^{2}=\left(\left(n_{1}-1\right) S_{\mathrm{i}}^{2}+\left(n_{2}-1\right) S_{2}^{2}\right) /$ $\left(n_{1}+n_{2}-2\right)$. Under the null hypothesis, $T(p) \sim t_{n_{1}}+n_{2}-2$ the $t$-distribution with $n_{1}+n_{2}-2$ degrees of freedom at each fixed 
point $p$. The type I error ( $\alpha$ level) for the multiple hypotheses testing would be

$$
\begin{aligned}
\alpha & =P\left(\text { reject at least one } H_{0}(p) \mid H_{0} \text { true }\right) \\
& =P\left(\sup _{p \in \partial \Omega} T(p)>h\right) \text { for some } h .
\end{aligned}
$$

The distribution of $\sup _{p \in \Omega} T(p)$ is asymptotically given as

$$
P\left(\sup _{p \in \partial \Omega} T(p)>h\right) \approx \sum_{d=0}^{2} \phi_{d}(\partial \Omega) \rho_{d}(h)
$$

where $\phi_{d}$ are the $d$-dimensional Minkowski functionals of $\partial \Omega$ and $\rho_{d}$ are the $d$-dimensional Euler characteristic (EC) density of $t$-field (Worsley, 1994). In order for the random field theory for multiple comparison to be valid, the data must be Gaussian and smooth. Heat kernel smoothed cortical thickness measurements tend to reasonably follow random field assumptions when large band width is used. Fig. 6 demonstrates that the data are increasingly Gaussian with larger band width. See Chung et al. (2003) for the demonstration of increasing Gaussianness using Lilliefors statistic. The Minkowski functionals are $\phi_{0}=2, \phi_{1}=$ $0, \phi_{2}=\mu(\partial \Omega) / 2=49,616 \mathrm{~mm}^{2}$, the half area of the template cortex $\partial \Omega$. The EC densities $\rho_{0}$ and $\rho_{2}$ are given by

$$
\begin{aligned}
& \rho_{0}(h)=\int_{h}^{\infty} \frac{\Gamma\left(\frac{d+1}{2}\right)}{(d \pi)^{1 / 2} \Gamma\left(\frac{d}{2}\right)}\left(1+\frac{x^{2}}{d}\right)^{-\frac{(\mathrm{d}+1)}{2}} \mathrm{~d} x, \\
& \rho_{2}(h)=\frac{\lambda}{(2 \pi)^{3 / 2}} \frac{\Gamma\left(\frac{d+1}{2}\right)}{\left(\frac{d}{2}\right)^{1 / 2} \Gamma\left(\frac{d}{2}\right)}\left(1+\frac{h^{2}}{d}\right)^{-\frac{(\mathrm{d}-1)}{2}} h,
\end{aligned}
$$

where $\lambda$ measures the smoothness of fields (Worsley et al., 1992). If we assume $\varepsilon$ to be a unit variance isotropic field, the smoothness of an isotropic random field can be defined as the covariance of derivative vector $\partial \varepsilon(x) / \partial x$, given by Cov $\partial \varepsilon(x) / \partial x=$ $\lambda I$. If $\varepsilon$ is the convolution of the Gaussian white noise with isotropic kernel $K_{\sigma}$, we can show that $\lambda=1 /\left(2 \sigma^{2}\right)$. In brain imaging the amount of smoothing $\sigma^{2}$ is usually expressed in terms of the full width at the half maximum (FWHM) of a smoothing kernel. Note that the FWHM of kernel $K_{\sigma}$ is $2 \sqrt{\ln 4} \sigma$. Conversely for given FWHM, the corresponding kernel is $K_{\mathrm{FWHM} /(2 \sqrt{\ln 4})}$. So in terms of FWHM, the smoothness of field is given as $\lambda=4 \ln 2$ / $\mathrm{FWHM}^{2}$. The FWHM is usually predetermined to match the extent of the signal size and we set it to be $30 \mathrm{~mm}$. The resulting corrected $P$ value map is given in Fig. 8.

We also removed the effect of age and total gray matter volume by setting up a general linear model (GLM) on cortical thickness $Y_{j}$ for subject $j$

$$
\begin{aligned}
Y_{j}(p)= & \lambda_{1}(p)+\lambda_{2}(p) \times \operatorname{age}_{j}+\lambda_{3}(p) \\
& \times \text { volume }_{j}+\beta(p) \times \text { group }_{j}+\varepsilon
\end{aligned}
$$

where dummy variable group is 1 for the autistic subjects and 0 for the normal subjects. Then, we test the group difference

$H_{0}: \beta(p)=0$ for all $p \in \partial \Omega$

vs.

$H_{1}: \beta(p) \neq 0$ for some $p \in \partial \Omega$

via the maxima of a $F$-field (Worsley, 1994).

\section{Results and discussion}

Image acquisition and processing were performed as described in Subjects and image processing, resulting in a cortical thickness map and the total gray matter volume (see Table 1) for each subject. The thickness measurements were then smoothed with the heat kernel of size $30 \mathrm{~mm}$ FWHM as described in Heat kernel smoothing and used to compute the corrected $P$ value maps for $t$ and $F$ statistics as discussed in Statistical analysis on cortical manifolds. First, we performed analysis without removing the effects of age and total gray matter volume to initially access the thickness pattern difference. The regions of thickness difference between two groups are assessed via the two-sample $t$ test with random field theory based $P$ value correction. The resulting corrected $P$ value map is given in the first two rows in Fig. 8. The first row is the $P$ value map projected onto the outer average surface while the second row is the $P$ value map projected onto the inner average surface. Blue (red) regions show statistically significant thickness reduction (increase) in the autistic group relative to the control group (thresholded at the corrected $P$ value $<0.1$ ) The right inferior orbital prefrontal cortex, the left superior temporal sulcus and the left occipito-temporal gyrus show dominant thickness decrease in the autistic group relative to the control group. The left superior temporal gyrus, the left middle temporal gyrus and the both left and right postcentral sulci show the thickness increase in the autistic group relative to the control groups, however, these regions turn out to be not very significant after removing the effect of age and total gray matter volume using general linear model (5). Note that the autistic subjects show relatively larger total gray matter volume: the mean gray matter volumes are $7.08 \pm 0.46 \mathrm{~mm}^{3}$ for the autistic group and $6.95 \pm 0.33 \mathrm{~mm}^{3}$ for the control group. After removing the effect of age and gray matter, the statistically significant regions of thickness decrease are detected at the right inferior orbital prefrontal cortex, the left superior temporal sulcus, and the left occipito-temporal gyrus.

Our results confirm some of previous voxel-based frontotemporal gray matter abnormality studies (Abell et al., 1999; Boddaert et al., 2004; Waiter et al., 2004) with some differences. We have compared our results to the voxel-based morphometry (VBM) results since no cortical thickness analysis has previously been performed with autistic subjects. Differences between our results and previous VBM results are expected since what we measure is the thickness of gray matter while VBM measures probabilistic gray matter volume. Also, our heat kernel smoothing utilizes the convoluted geometry of the cortex while 3D Gaussian kernel smoothing used in VBM does not. Also, most previous autism anatomical studies did not remove the effect of age while our analysis did. This may give fewer false positive findings as illustrated in Fig. 8. Our results are consistent with our previous VBM study applied to the sagittal cross-section of the corpus callosum to detect abnormal inter-hemispheric connectivity (Chung et al., 2003). It was shown that there is less white matter concentration in the rostrum and the splenium in the autistic group. The orbital frontal cortex projects through the rostrum while inferior temporal cortex projects through the splenium (Hardan et al., 2000) so it was conjectured in Chung et al. (2003) that we would find some abnormality in these cortical regions.

Using VBM, Abell et al. (1999) found decreased gray matter volume in the right paracingulate sulcus, the left inferior frontal gyrus and the left occipito-temporal junction in the autistic group relative to the control group (uncorrected $P$ value $<0.001$ ). 


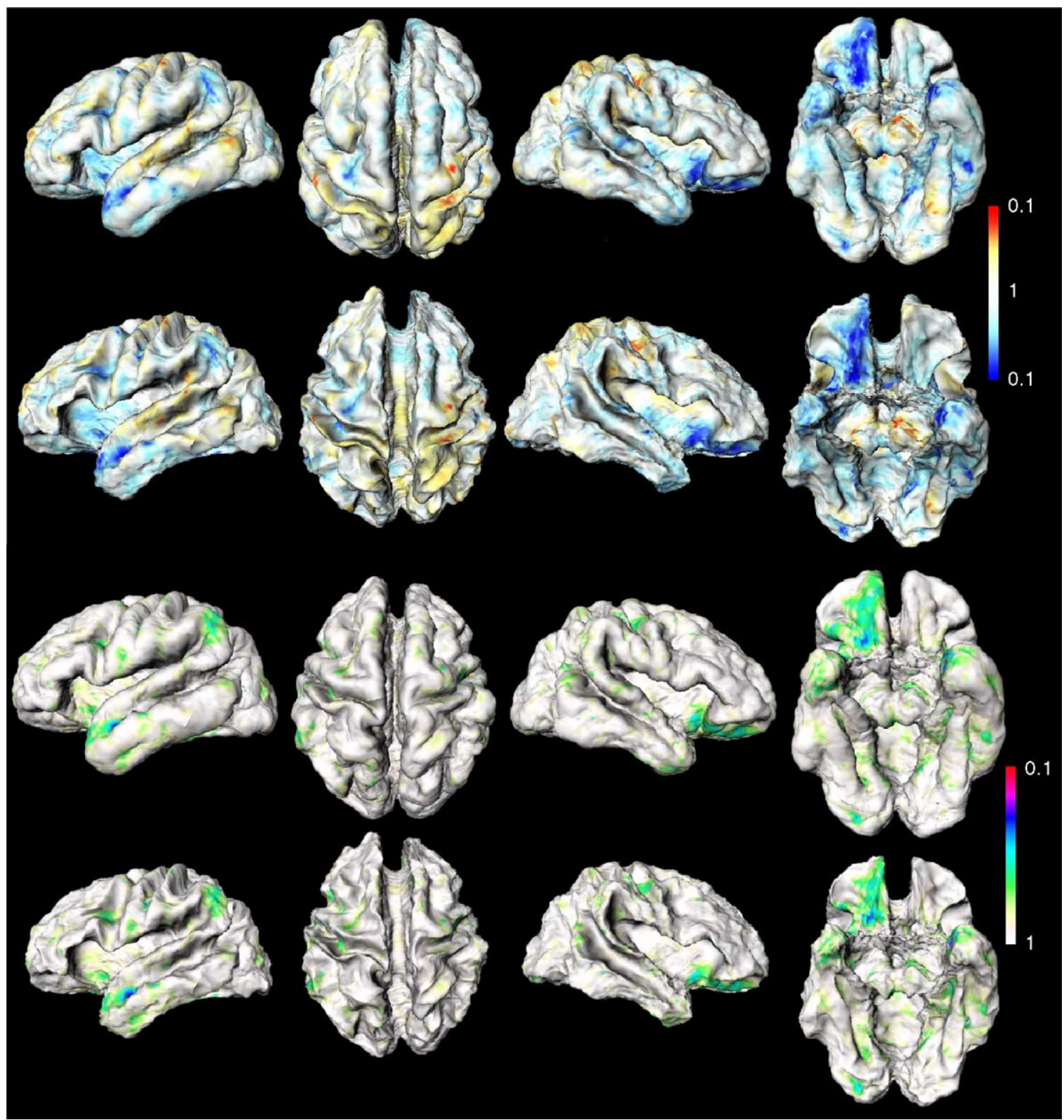

Fig. 8. Corrected $P$ value maps projected onto the average outer (1st and 3rd rows) and inner surfaces (2nd and 4th rows). First two rows: two-sample $t$ test results. Red is the regions of thicker gray matter while blue is thinner gray matter in the autistic subjects. Last two rows: $F$ test results removing the effect of age and relative gray matter volume difference. $F$ test results shows relatively asymmetric thickness difference between two groups. Comparing two $P$ value maps, it can be seen that the thicker gray matter region is largely due to the effect of age and gray matter volume difference.

Although we did not detect statistically significant decreased thickness in the left inferior frontal regions, there is a tendency for it to be relatively thinner as shown in Fig. 8. They also found increased gray matter volume in the left middle temporal gyrus and the right inferior temporal gyrus. Their analysis did not remove the effect of age so their findings more closely resemble our twosample $t$ test results than the $F$ test results. Using the same VBM, Boddaert et al. (2004) found bilaterally significant decreases of gray matter concentration in superior temporal sulcus but did not detect any other abnormality outside the temporal lobes. They did not remove the effect of age. The results contradict our result since our result shows more widespread asymmetric thickness abnormality in both the inferior prefrontal cortex and the superior temporal sulcus. In the future, more thorough hemispheric asymmetry analysis is needed. Using VBM, Waiter et al. (2004) detected increased gray matter volume in the left inferior frontal gyrus and left superior temporal gyrus that only just failed to reach the corrected $P$ value of 0.1 . They also did not remove the effect of age in their analysis.

\section{Acknowledgments}

Authors with to thank Arnaud Cachia at the Service Hospitalier Frédéric Joliot, CEA, Orsay, France for providing the traces of the central and superior temporal sulcal fundi used in producing Fig. 3. Authors also wish to thank Keith Worsley of the McGill University, Kam Tsui and Shijie Tang of the Department of 
Statistics, University of Wisconsin-Madison and Tulaya Limpiti of the Department of Electrical Engineering, University of WisconsinMadison for valuable discussions on heat kernel smoothing. This research is funded by Wisconsin Alumni Research Foundation (WARF), MIMH U54 MH066398, NIH U19 HD035476 and by a NARSAD Distinguished Investigator Award to RJD.

\section{References}

Abell, F., Krams, M., Ashburner, J., Passingham, R., Friston, K., Frackowiak, R., Happe, F., Frith, C., Frith, U., 1999. The neuroanatomy of autism: a voxel-based whole brain analysis of structural scans. NeuroReport 10, 1647-1651.

Andrade, A., Kherif, F., Mangin, J., Worsley, K.J., Paradis, A., Simon, O., Dehaene, S., Le Bihan, D., Poline, J-B., 2001. Detection of fMRI activation using cortical surface mapping. Hum. Brain Mapp. 12, 79-93.

Ashburner, J., Friston, K., 2000. Voxel-based morphometry-the methods. NeuroImage 11, 805-821.

Berline, N., Getzler, E., Vergne, M., 1991. Heat kernels and dirac operators. Springer-Verlag, New York.

Boddaert, N., Chabane, N., Gervais, H., Good, C.D., Bourgeois, M., Plumet, M.H., Barthelemy, C., Mouren, M.C., Artiges, E., Samson, Y., Brunelle, F., Frackowiak, R.S., Zilbovicius, M., 2004. Superior temporal sulcus anatomical abnormalities in childhood autism: a voxel-based morphometry MRI study. NeuroImage 23, 364-369.

Cachia, A., Mangin, J.-F., Riviére, D., Kherif, F., Boddaert, N., Andrade, A., Papadopoulos-Orfanos, D., Poline, J.-B., Bloch, I., Zilbovicius, M., Sonigo, P., Brunelle, F., Régis, J., 2003a. A primal sketch of the cortex mean curvature: a morphogenesis based approach to study the variability of the folding patterns. IEEE Trans. Med. Imaging 22, 754-765.

Cachia, A., Mangin, J.-F., Riviére, D., Papadopoulos-Orfanos, D., Kherif, F., Bloch, I., Régis, J., 2003b. A generic framework for parcellation of the cortical surface into gyri using geodesic Voronoï diagrams. Med. Image Anal. 7, 403-416.

Chung, M.K., 2004. Heat kernel smoothing and its application to cortical manifolds. Technical Report 1090. Department of Statistics, University of Wisconsin, Madison.

Chung, M.K., Taylor, J., 2004. Diffusion smoothing on brain surface via finite element method. Proceedings of IEEE International Symposium on Biomedical Imaging (ISBI).

Chung, M.K., Worsley, K.J., Robbins, S., Paus, P., Taylor, J., Giedd, J.N., Rapoport, J.L., Evans, A.C., 2003. Deformation-based surface morphometry applied to gray matter deformation. NeuroImage 18 , $198-213$.

Collins, D.L., Neelin, P., Peters, T.M., Evans, A.C., 1994. Automatic 3D intersubject registration of MR volumetric data in standardized Talairach space. J. Comput. Assist. Tomogr. 18, 192-205.

Dale, A.M., Fischl, B., 1999. Cortical surface-based analysis: I. Segmentation and surface reconstruction. NeuroImage 9, 179-194.

Davatzikos, C., 1997. Spatial transformation and registration of brain images using elastically deformable models. Comput. Vision Image Underst. 66, 207-222.

Davatzikos, C., Bryan, R.N., 1995. Using a deformable surface model to obtain a shape representation of the cortex. Proc. IEEE Int. Conf. Comput. Vision, 2122-2127.

Fischl, B., Dale, A.M., 2000. Measuring the thickness of the human cerebral cortex from magnetic resonance images. Proc. Natl. Acad. Sci. $97,11050-11055$.

Fischl, B., Sereno, M.I., Tootell, R., Dale, A.M., 1999. High-resolution intersubject averaging and a coordinate system for the cortical surface. Hum. Brain Mapp. 8, 272-284.
Hardan, A.Y., Minshew, N.J., Keshavan, M.S., 2000. Corpus callosum size in autism. Neurology 55, 1033-1036.

Jones, S.E., Buchbinder, B.R., Aharon, I., 2000. Three-dimensional mapping of cortical thickness using Laplace's equation. Hum. Brain Mapp. 11, 12-32.

Joshi, S.C., Wang, J., Miller, M.I., Van Essen, D.C., Grenander, U., 1995. On the differential geometry of the cortical surface. Vision Geom. IV 2573, 304-311.

Kabani, N., Le Goualher, G., MacDonald, D., Evans, A.C., 2000. Measurement of cortical thickness using an automated 3-D algorithm: a validation study. NeuroImage 13, 375-380.

Kiebel, S., Friston, K.J., 2002. Anatomically informed basis functions in multisubject studies. Hum. Brain Mapp. 16, 36-46.

Koenderink, J.J., 1984. The structure of images. BCyber 50, 363-370.

Kollakian, K., 1996. Performance analysis of automatic techniques for tissue classification in magnetic resonance images of the human brain. Master's thesis, Concordia Univ., Canada.

Lerch, J., Evans, A.C., 2005. Cortical thickness analysis examined through power analysis and a population simulation. NeuroImage 24, $163-173$.

MacDonald, J.D., Kabani, N., Avis, D., Evans, A.C., 2000. Automated 3-D extraction of inner and outer surfaces of cerebral cortex from MRI. NeuroImage 12, 340-356.

Miller, M.I., Massie, A.B., Ratnanather, J.T., Botteron, K.N., Csernansky, J.G., 2000. Bayesian construction of geometrically based cortical thickness metrics. NeuroImage 12, 676-687.

Liu, T., Shen, D., Davatzikos, C., 2004. Deformable registration of cortical structures via hybrid volumetric and surface warping. NeuroImage 22, $1790-1801$.

Luders, E., Rex, D.E., Narr, K.L., Woods, R.P., Janke, L., Thompson, P.M., Mazziotta, J.C., Toga, A.W., 2003. Relationships between sulcal asymmetries and corpus callosum size: gender and handedness effects. Cereb. Cortex 13, 1084-1093.

Robbins, S.M., 2003. Anatomical standardization of the human brain in euclidean 3-space and on the cortical 2-manifold. PhD Thesis, School of Computer Science, McGill University, Montreal, QC, Canada.

Rosenberg, S., 1997. The Laplacian on a Riemannian manifold. Cambridge Univ. Press.

Sled, J.G., Zijdenbos, A.P., Evans, A.C., 1988. A nonparametric method for automatic correction of intensity nonuniformity in MRI data. IEEE Trans. Med. Imaging 17, 87-97.

Thompson, P.M., Toga, A.W., 1996. A surface-based technique for warping 3-dimensional images of the brain. IEEE Trans. Med. Imaging $15,1-16$

Thompson, P.M., Hayashi, K.M., Sowell, E.R., Gogtay, N., Giedd, J.N., Rapoport, J.L., de Zubicaray, G.I., Janke, A.L., Rose, S.E., Semple, J., Doddrell, D.M., Wang, Y.L., van Erp, T.G.M., Cannon, T.D., Toga, A.W. in press. Mapping cortical change in alzheimer s disease, brain development, and schizophrenia, NeuroImage 23S, 2-18.

Van Essen, H., Drury, A., Joshi, S., Miller, M.I., 1998. Functional and structural mapping of human cerebral cortex: solutions are in the surfaces. Proc. Natl. Acad. Sci. 95 (3), 788-795.

Waiter, G.D., Williams, J.H., Murray, A.D., Gilchrist, A., Perrett, D.I., Whiten, A., 2004. A voxel-based investigation of brain structure in male adolescents with autistic spectrum disorder. NeuroImage 22, $619-625$

Worsley, K.J., 1994. Local maxima and the expected Euler characteristic of excursion sets of $\chi 2, F$ and $t$ fields. Adv. Appl. Probab. 26, 13-42.

Worsley, K.J., Marrett, S., Neelin, P., Evans, A.C., 1992. A threedimensional statistical analysis for CBF activation studies in human brain. J. Cereb. Blood Flow Metab. 12, 900-918.

Zijdenbos, A.P., Jimenez, A., Evans, A.C., 1998. Pipelines: large scale automatic analysis of 3D brain data sets. NeuroImage 7S, 783 . 